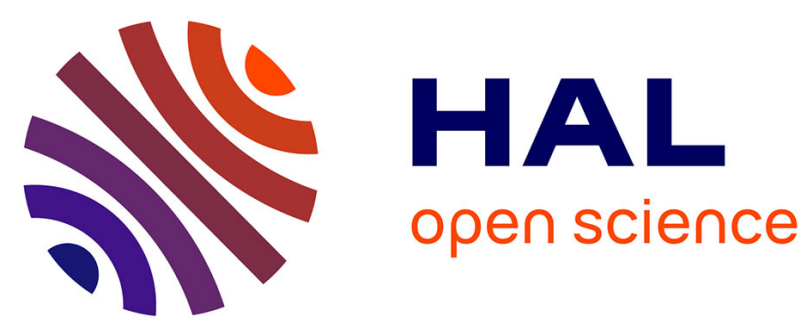

\title{
Spin-Torque Influence on the High-Frequency Magnetization Fluctuations in Magnetic Tunnel Junctions
}

S. Petit-Watelot, C. Baraduc, C. Thirion, U. Ebels, Y Liu, M. Li, P. Wang, B. Dieny

\section{To cite this version:}

S. Petit-Watelot, C. Baraduc, C. Thirion, U. Ebels, Y Liu, et al.. Spin-Torque Influence on the HighFrequency Magnetization Fluctuations in Magnetic Tunnel Junctions. Physical Review Letters, 2007, 98 (7), pp.077203. 10.1103/PhysRevLett.98.077203 . hal-01636635

\section{HAL Id: hal-01636635 \\ https://hal.univ-lorraine.fr/hal-01636635}

Submitted on 16 Nov 2017

HAL is a multi-disciplinary open access archive for the deposit and dissemination of scientific research documents, whether they are published or not. The documents may come from teaching and research institutions in France or abroad, or from public or private research centers.
L'archive ouverte pluridisciplinaire HAL, est destinée au dépôt et à la diffusion de documents scientifiques de niveau recherche, publiés ou non, émanant des établissements d'enseignement et de recherche français ou étrangers, des laboratoires publics ou privés. 


\title{
Spin-Torque Influence on the High-Frequency Magnetization Fluctuations in Magnetic Tunnel Junctions
}

\author{
S. Petit, ${ }^{1}$ C. Baraduc, ${ }^{1}$ C. Thirion,,${ }^{1}$ U. Ebels,${ }^{1}$ Y. Liu, ${ }^{2} \mathrm{M} . \mathrm{Li},{ }^{2}$ P. Wang, ${ }^{2}$ and B. Dieny ${ }^{1}$ \\ ${ }^{1}$ SPINTEC, URA 2512 CEA/CNRS, CEA/Grenoble, 38054 Grenoble Cedex 9, France \\ ${ }^{2}$ Headway Technologies, 678 Hillview Drive, Milpitas California 95035, USA
}

(Received 24 October 2006; published 12 February 2007)

\begin{abstract}
Voltage noise measurements were performed in the 3-7 GHz frequency range on magnetic tunnel junctions biased with a dc current. Magnetic noise associated with ferromagnetic resonance excitations is either amplified or reduced depending on the direction of the bias current. This effect is interpreted as the influence of spin transfer torque on the magnetization fluctuations and described using Gilbert dynamics equation including spin transfer torque and effective field terms.
\end{abstract}

In magnetic multilayers, electron transport properties are governed by the relative magnetization orientation. This effect is at the origin of giant magnetoresistance or tunnel magnetoresistance and is widely used in spintronics devices. Moreover, a spin-polarized current reciprocally interacts with the magnetization through direct transfer of spin angular momentum. This interaction was theoretically addressed ten years ago independently by Slonczewski and Berger [1,2]. Since then, the concept of spin transfer torque attracted a considerable interest; for example, it was experimentally demonstrated that a dc current could induce magnetization reversal in a ferromagnetic nanostructure, provided the current density is larger than a critical value $j_{c}$ [3]. The influence of spin transfer torque on the magnetization dynamics has also been widely studied in relation to potential applications such as rf oscillators. In particular, electrical measurements at microwave frequency provided evidence of steady state precession induced by a dc current of the order of the critical current [4,5].

In this Letter we show that spin transfer torque effects are detected at current densities much below the critical value $j_{c}$ in submicron sized magnetic tunnel junctions. By developing a very sensitive noise experiment in the $\mathrm{GHz}$ frequency range, we have observed a significant influence of spin transfer torque on thermally activated ferromagnetic resonance (FMR) excitations at relatively low current bias $\left(j_{c} / 20 \leq j \leq j_{c} / 4\right)$ : the magnetization fluctuations at the FMR are either enhanced or quenched depending on the dc-current direction. In fact, evidences of spin transfer torque without dc-current bias have been obtained, but with magnetization driven by an ac-current excitation [6]. However, in our study the sample is not subjected to any rf excitation. To our knowledge our experiment provides the first evidence of spin transfer torque in the nearequilibrium regime.

Here the influence of the spin-torque term on magnetic fluctuations is modeled using the macrospin approximation. As we study low current effects, the system is near static equilibrium and is well described by the linear response theory. Therefore, we develop a model following this scheme: (i) the susceptibility is calculated by taking into account spin transfer effects; (ii) the fluctuation spectrum is expressed according to the fluctuation-dissipation theorem. Let us consider a continuous ferromagnetic film with the $x$ axis perpendicular to the plane, subjected to a static magnetic field applied along the $z$ direction. Magnetization dynamics is assumed to follow the Landau-Lifschitz-Gilbert equation modified with the spin transfer terms [7]:

$$
\begin{aligned}
\frac{d \vec{M}}{d t}= & -\gamma \vec{M} \times \vec{H}_{\mathrm{eff}}+\frac{\alpha}{M_{s}} \vec{M} \times \frac{d \vec{M}}{d t} \\
& -\frac{\gamma a_{j}}{M_{s}} \vec{M} \times(\vec{M} \times \vec{P})-\gamma b_{j} \vec{M} \times \vec{P},
\end{aligned}
$$

where $\gamma$ is the gyromagnetic ratio, $\alpha$ the Gilbert damping constant, $M_{s}$ the saturation magnetization, and $\vec{H}_{\text {eff }}=$ $\vec{H}_{b}+4 \pi M_{x} \vec{e}_{x}+\vec{h}$ is the sum of the applied $\vec{H}_{b}$, demagnetizing and weak excitation $\vec{h}$ field. The third term in the right-hand side of the equation is the Slonczewski spintorque term where $a_{j}$ is proportional to the current density $j$, and $\vec{P}$ is the unit vector of incoming electrons polarization. The last term is the current driven interlayer exchange coupling where $b_{j}$ is proportional to the current density. The influence of this fieldlike term will be checked by comparing results with $b_{j}$ set to zero and nonzero values. The susceptibility matrix is analytically derived from the dynamics equation assuming a linear harmonic response to the excitation field. The dominant term is $\chi_{y y}$ whose real and imaginary parts are given below:

$$
\begin{aligned}
& \chi_{y y}^{\prime}=\gamma M_{s} \frac{\gamma H^{\prime}\left[\omega_{0}^{2}-\left(1+\alpha^{2}\right) \omega^{2}\right]+\alpha \Delta \omega^{2}}{\left[\omega_{0}^{2}-\left(1+\alpha^{2}\right) \omega^{2}\right]^{2}+\Delta^{2} \omega^{2}} \\
& \chi_{y y}^{\prime \prime}=\gamma M_{s} \omega \frac{\Delta \gamma H^{\prime}-\alpha\left[\omega_{0}^{2}-\left(1+\alpha^{2}\right) \omega^{2}\right]}{\left[\omega_{0}^{2}-\left(1+\alpha^{2}\right) \omega^{2}\right]^{2}+\Delta^{2} \omega^{2}}
\end{aligned}
$$

with the following notations: 


$$
\begin{gathered}
H_{b}^{\prime}=H_{b}+b_{j}, \quad H^{\prime}=H_{b}^{\prime}+4 \pi M_{s}, \\
\omega_{0}^{2}=\gamma^{2}\left[H^{\prime} H_{b}^{\prime}+a_{j}^{2}\right], \\
\Delta / \gamma=\alpha\left[4 \pi M_{s}+2 H_{b}+2 b_{j}\right]+2 a_{j},
\end{gathered}
$$

where $\Delta$ is the resonance peak linewidth. To a first order approximation ( $\alpha \ll 1$ and $a_{j} \ll M_{s}$ ), the resonance frequency is

$$
\omega_{r}^{2} \approx \gamma^{2}\left[H^{\prime} H_{b}^{\prime}-\frac{\alpha^{2}}{2} H^{\prime}\left(H^{\prime}+3 H_{b}^{\prime}\right)-\alpha\left(2 H^{\prime}+H_{b}^{\prime}\right) a_{j}\right] .
$$

Magnetization fluctuations induce resistance noise due to the magnetoresistive effect. Assuming that the free layer magnetization fluctuates only within the layer plane $(y, z)$ and that the out-of-plane magnetization component $\left(M_{x}\right)$ can be neglected, the noise voltage can be expressed as follows when the external field is applied at an angle $\theta$ away from the reference layer direction:

$$
\delta V=I \delta R=\frac{I \Delta R}{2 M_{s}} \delta m_{y} \sin (\theta),
$$

where $\delta m_{y}$ is the fluctuating magnetization transverse component and $\Delta R$ the maximum magnetoresistance. According to the fluctuation-dissipation theorem [8,9], the power spectral density of the transverse magnetization fluctuations is expressed by the following expression:

$$
S_{m_{y}}=\frac{4 k T}{V} \frac{\chi_{y y}^{\prime \prime}}{\omega} .
$$

We cross-checked our result by calculating the fluctuating field $\left(h_{x}, h_{y}\right)$ that acts as Langevin forces on the magnetization. The fluctuating field power spectrum is a function of the inverse susceptibility matrix. Calculations with the susceptibility expression including spin-torque terms give the usual isotropic white spectrum of the fluctuating field at equilibrium [10].

From numerical evaluation of the noise spectra, using Eq. (3) with $b_{j}=0$, we observe that the noise peak corresponding to FMR excitations either increases or decreases depending on the current flow direction. This effect is related to the (de)-stabilization of the equilibrium magnetization by the spin transfer torque. When the FMR excitations peak increases, we observe a slight shift of the peak frequency towards higher frequency. This may be interpreted as a smaller effective damping due to the spin transfer torque. With $b_{j} \neq 0$, we observe that the computed noise peak shifts now towards low frequency without any significant change of amplitude. In fact, from Eq. (4) it is straightforward to estimate the dependence of the resonance frequency upon the applied current: $d \omega_{r} / d j \propto$ $-2 \alpha a_{j} / j+b_{j} / j$. Since $\alpha \ll 1$ we see that the fieldlike term has an opposite and much stronger effect than the $a_{j}$ term on the resonance frequency. The computed noise spectrum characteristics and their dependence upon $a_{j}$ and $b_{j}$ will be discussed and compared with our experimental data.

In order to check our model, we studied magnetic tunnel junctions with 20\% MR ratio developed for readhead applications with the following multilayer composition: $\mathrm{PtMn}_{(20)} / \mathrm{CoFe}_{(3)} / \mathrm{Ru}_{(0.7)} / \mathrm{CoFe}_{(2.5)} / \mathrm{Al}_{2} \mathrm{O}_{3(0.7)} / \mathrm{CoFe}_{(1)} /$ $\mathrm{NiFe}_{(3)}$, where the subscript corresponds to the layer thickness in $\mathrm{nm}$. After deposition, the stack is etched into a pillar with a $300 \mathrm{~nm}$ diameter circular cross section. It is connected at the top to a large metallic lead and at the bottom to a narrow NiFe lead acting as a shield. Voltage noise measurements were performed in the $\mathrm{GHz}$ frequency range as a function of the external magnetic field and a dc current applied to the sample. Noise spectra were measured using a low noise preamplifier and a spectrum analyzer. The data were subsequently corrected from the noise induced by the measurement setup itself and from the transfer function of the line. First, the noise power spectra at zero bias current were substracted from those measured at nonzero bias. This removes the background Johnson noise as well as the amplifier noise. Then the data were divided by the gain function of the preamplifier $G(f)$ and corrected from the impedance mismatch of the sample. For that purpose, the complex impedance of the sample was measured beforehand with a vector network analyzer and deembedding was performed in order to quantify the junction resistance, the lead resistance, and parasitic inductance and capacitance. Note that the capacitance formed by the facing areas between the top and bottom leads is responsible for a signal cutoff at about $8 \mathrm{GHz}$. Finally, in order to extract the magnetic noise contribution, all power noise spectra were normalized by $[I \Delta R(I)]^{2}$. Division by $I$ gives the resistance noise, then normalization by $\Delta R(I)=$ $R_{A P}(I)-R_{P}(I)$ corrects from the well-known fact that magnetoresistance of tunnel junctions decreases with current bias.

Voltage noise was measured both in the parallel $(P)$ and antiparallel $(A P)$ states as a function of magnetic field and dc-current bias. In the $1-6 \mathrm{GHz}$ frequency range, the magnetic noise spectra show series of peaks that we ascribe to thermally excited ferromagnetic resonance. Since the observed resonance frequency is close to the bulk fundamental frequency, we conclude to a slight field misalignment, otherwise the resonance would occur at twice the fundamental frequency. By comparing the observed and computed peak amplitude, the misalignment angle does not exceed $3^{\circ}$. In the noise spectrum two peaks corresponding to two distinct modes are sufficiently well defined to be examined in detail. We observe that the peaks decrease in amplitude and shift to higher frequencies upon increasing external magnetic field (Fig. 1). This is consistent with the Kittel formula: the frequency squared is linear with the applied field for both peaks. It is assumed that both modes correspond to quantified volume modes due to their iden- 


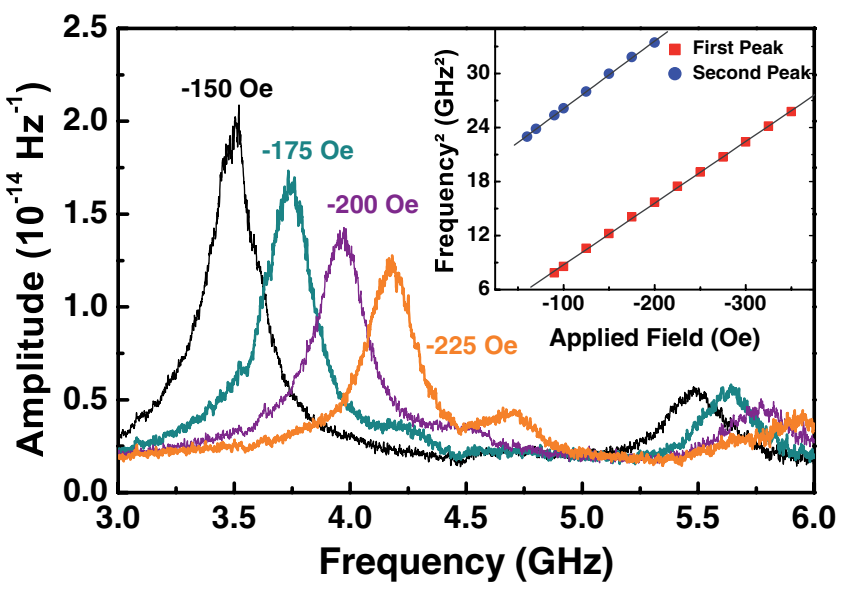

FIG. 1 (color online). Normalized noise spectra at various applied magnetic fields. The peak frequency of each mode verifies the Kittel formula with identical slope (see inset).

tical slopes [11]. The slope value corresponds to a saturation magnetization of $8950 \mathrm{Oe}$, that is $27 \%$ smaller than the bulk value. This characteristic has been observed in other FMR experiments on nanostructures [12,13]. Concerning the noise amplitude, it decreases with increasing field and fits well a $1 / H$ law derived from Eq. (3). This behavior can be explained by the higher energy cost of fluctuations under larger magnetic field.

In contrast to the field dependence of the power spectrum, the evolution with current is quite different, revealing a huge variation of the peak amplitude with little frequency shift. The most striking feature is a pronounced asymmetry as a function of the current sign. The magnetic noise is either amplified or quenched depending on the bias current direction (Fig. 2). In the $A P$ state $\left(H<-H_{c}\right)$, we observe that the peak amplitude increases by $300 \%$ when the negative bias current is increased. When the current sign is reversed, the peak decreases and finally vanishes with

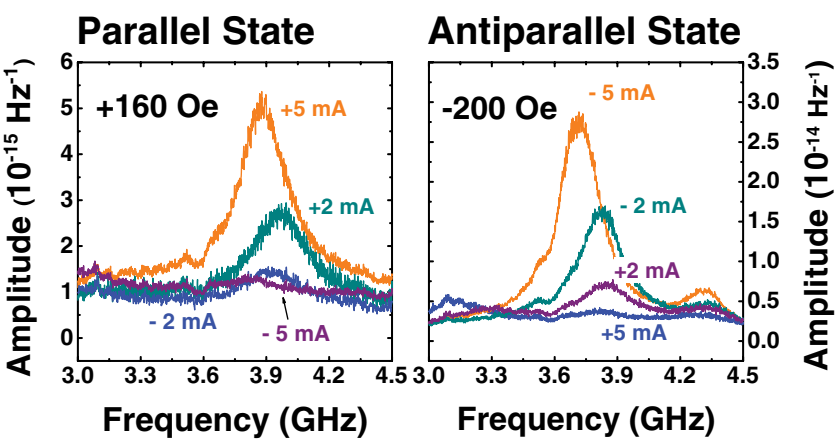

FIG. 2 (color online). Normalized noise spectra in the $P$ and $A P$ states as a function of the dc bias current. Magnetic noise is either reduced or enhanced depending on the sign of the bias current. The effect is reversed when changing from $P$ to $A P$ state. Since the hysteresis loop of the free layer is shifted by $-20 \mathrm{Oe}$, the $P$ and $A P$ states correspond to perfectly symmetric states with respect to the applied field. increasing current. Moreover, in the $P$ state $\left(H>H_{c}\right)$, the effect of the current sign is reversed: the peak grows with positive current and lowers with negative current. This behavior suggests a pronounced influence of the spin transfer torque on the noise spectrum. At negative current, in our experiment, electrons flow from the reference layer to the free layer. Spin transfer torque then tends to favor the parallel magnetic configuration. It is observed that the magnetic fluctuations are consistently quenched in the $P$ state and enhanced in the $A P$ state. For positive current, electrons flow from the free layer to the reference layer. Spin transfer torque now tends to favor the antiparallel magnetic configuration. Therefore, the effect of spin transfer torque on magnetic fluctuations is reversed compared to the negative current case. Let us stress that the applied currents $\left[\left(1.4-8.4 \times 10^{6} \mathrm{~A} / \mathrm{cm}^{2}\right)\right]$ are much smaller than the estimated threshold value $\left(4 \times 10^{7} \mathrm{~A} / \mathrm{cm}^{2}\right)$, so the regions of the current-field phase diagram that are studied are near-equilibrium states, far from any transition to steady state precession.

Let us now examine the spin-torque influence on magnetic noise at the dominant resonance, i.e., for the largest peak in the noise spectrum. According to our model the peak amplitude is expected to diverge at the critical current $I_{c}$ which is defined when $\Delta=0$. The critical current is the limit value for which fluctuations diverge and steady state precession occurs. Experimentally $I_{c}$ is about $20 \pm 5 \mathrm{~mA}$ which corresponds to a current density of about $4 \times$ $10^{7} \mathrm{~A} / \mathrm{cm}^{2}$ (Fig. 3). The corresponding peak linewidth $\Delta$ decreases (increases) when the peak amplitude increases (decreases) and shows a linear dependence consistent with our model. The linear extrapolation of the linewidth to zero yields the same value of the critical current as given above. The value of the spin-torque term coefficient $\left(a_{j} / j\right)$ is then extracted from the measured critical current and is approximately $7 \mathrm{Oe} / \mathrm{mA}\left(5 \times 10^{-6} \mathrm{Oe} \mathrm{A}^{-1} \mathrm{~cm}^{2}\right)$ assuming that the Gilbert damping term $\alpha$ is equal the bulk value: $\alpha=$ 0.031. In the above evaluation, $b_{j}$ has not been considered since it has a minor influence on the amplitude and the divergence. Thus it does not alter the value of $a_{j}$ deter-
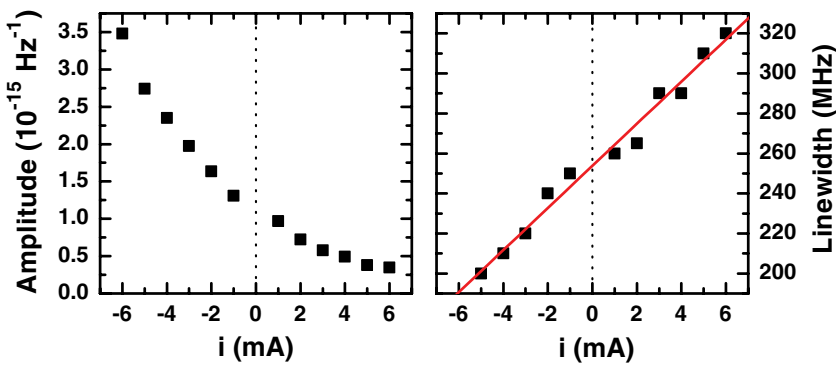

FIG. 3 (color online). Peak amplitude (left) and linewidth (right) as a function of the current bias in the $A P$ state $(-200 \mathrm{Oe})$. The critical current is estimated both from the amplitude divergence and from the linewidth extrapolation to zero. Both methods lead to $20 \pm 5 \mathrm{~mA}$. 


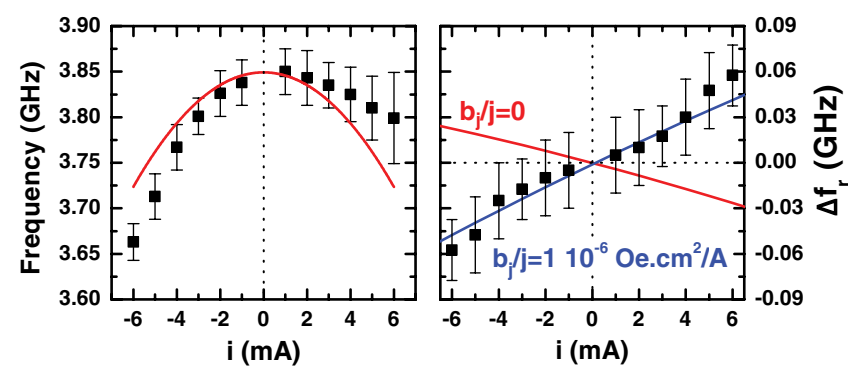

FIG. 4 (color online). Left: peak frequency as a function of the applied current in the $A P$ state $(-200 \mathrm{Oe})$. The parabola (solid line) corresponds to the frequency shift due to Joule heating. Right: shift of the resonance frequency corrected from the thermal effect. The fieldlike term $b_{j}$ cannot be neglected in order to have a good agreement between our model and the experimental data.

mined experimentally. However, from the model, we know that $b_{j}$ has a pronounced influence on the peak frequency dependence upon applied current; the slope of the peak frequency versus applied current may even change sign when considering $b_{j}$.

In the experiment, we observe a shift to lower frequency for both negative and positive currents, with a pronounced asymmetry (Fig. 4). This behavior is fitted by the sum of a linear and a quadratic term: $f-f_{0}=\kappa_{1} I+\kappa_{2} I^{2}$. The parabolic contribution may be ascribed to Joule heating. We checked in a separate experiment that the peak frequency shifts to lower values when temperature increases. This thermal effect has already been reported [12]. The mean value of the frequency shift roughly corresponds to a temperature increase of $40^{\circ} \mathrm{C}$ for a $5 \mathrm{~mA}$ current bias. When the peak frequency is corrected from this thermal effect we find that the frequency decreases for negative currents when the peak amplitude increases (Fig. 4). This behavior cannot be explained when considering only the spin-torque term $a_{j}$ since our model shows that it produces the opposite effect. Therefore, we propose to include the fieldlike term $b_{j}$ in order to reproduce the observed frequency shift as predicted by the model. We obtain a good agreement between experimental data and our calculations when using $b_{j} / j=1 \times 10^{-6} \mathrm{Oe} \mathrm{A}^{-1} \mathrm{~cm}^{2}$ which is of the order of $a_{j} / 5$. This value approximately corresponds to $1.5 \mathrm{Oe} / \mathrm{mA}$. It is relatively small and therefore might also be attributed to a possible contribution of the Oersted field caused by an asymmetric current flow through the pillar. However, experimental results are reproducible when the current is injected in the junction from another lead corresponding to a $\pi / 2$ rotation of the in-plane current lines. Therefore, our observation rather supports the idea of a fieldlike term in magnetic tunnel junctions, as it was already reported in a different experiment [6]. Moreover, our estimation of the ratio between $a_{j}$ and $b_{j}$ is consistent with theoretical calculations for magnetic tunnel junctions [14].

In summary, we have studied voltage noise of magnetic tunnel junctions in the $\mathrm{GHz}$ frequency range and observed that thermal magnetic fluctuations are either reduced or enhanced by the dc-current bias depending on its sign. We ascribe this effect to the influence of spin transfer torque on ferromagnetic resonance excitations depending on whether it favors or disfavors the equilibrium magnetic configuration. The experimental data are in good agreement with our model. In particular, we have shown that it is possible to extract the critical current and thus the spin-torque term $a_{j}$ from near-equilibrium effects. Finally, we suggest that a significant fieldlike term has to be taken into account for magnetic tunnel junctions.

We acknowledge A. Slavin and S. Demokritov for interesting discussions about this work. We also thank A. Schuhl for a careful reading of this manuscript. This work was partly supported by the European Commission under the RTN DYNAMICS (HPRN-CT-2002-00289) and SPINSWITCH (MRTN-CT-2006-035327) projects.

[1] L. Berger, Phys. Rev. B 54, 9353 (1996).

[2] J. Slonczewski, J. Magn. Magn. Mater. 159, L1 (1996).

[3] J. A. Katine, F. J. Albert, R. A. Buhrman, E. B. Myers, and D. C. Ralph, Phys. Rev. Lett. 84, 3149 (2000).

[4] S. Kiselev, J. Sankey, I. Krivorotov, N. Emley, R. Schoelkopf, R. Buhrman, and D. Ralph, Nature (London) 425, 380 (2003).

[5] W.H. Rippard, M. R. Pufall, S. Kaka, S. E. Russek, and T. J. Silva, Phys. Rev. Lett. 92, 027201 (2004).

[6] A. Tulapurkar, Y. Suzuki, A. Fukushima, H. Maehara, K. Tsunekawa, D. Djayaprawira, N. Watanabe, and S. Yuasa, Nature (London) 438, 339 (2005).

[7] S. Zhang, P. M. Levy, and A. Fert, Phys. Rev. Lett. 88, 236601 (2002).

[8] N. Smith, J. Appl. Phys. 90, 5768 (2001).

[9] N. Smith, J. Appl. Phys. 92, 3877 (2002).

[10] W. Brown, Phys. Rev. 130, 1677 (1963).

[11] G. Gubbiotti, G. Carlotti, T. Okuno, T. Shinjo, F. Nizzoli, and R. Zivieri, Phys. Rev. B 68, 184409 (2003).

[12] N. Stutzke, S. L. Burkett, and S. E. Russek, Appl. Phys. Lett. 82, 91 (2003).

[13] V. Synogatch, N. Smith, and J. Childress, J. Appl. Phys. 93, 8570 (2003).

[14] A. Manchon, N. Ryzhanova, N. Strelkov, A. Vedyayev, and B. Dieny, Special issue on Spin Electronics, J. Phys.: Condens. Matter (to be published). 